\section{Avaliação de programas comunitários de atividade física no Brasil: uma revisão de escopo}

\author{
Evaluation of community physical activity \\ programs in Brazil: a scoping review
}

\author{
Evaluación de programas comunitarios de \\ actividad física en Brasil: una revisión \\ de alcance
}

Alanna Gomes da Silva 1
Elton Junio Sady Prates 1
Deborah Carvalho Malta 1

doi: $10.1590 / 0102-311 \times 00277820$

\section{Resumo}

Os programas comunitários de atividade física foram criados para incentivar e aumentar esta atividade na população brasileira e promover hábitos de vida saudável. O Ministério da Saúde investiu na avaliação desses programas e consolidou parcerias que favoreceram a construção de importantes marcos sobre o tema. Este estudo objetivou identificar e sintetizar as evidências científicas sobre as abordagens e resultados das avaliações realizadas no Programa Academia da Saúde e Programa Academia da Cidade. Trata-se de uma revisão de escopo baseada na metodologia do Instituto Joanna Briggs. Utilizaram-se as bases MEDLINE via PubMed, LILACS, Scopus e Cochrane, o site do Programa Academia da Saúde, o Catálogo de Teses e Dissertações da Coordenação de Aperfeiçoamento de Pessoal de Nivel Superior e a Biblioteca Digital Brasileira de Teses e Dissertações. Incluíram-se estudos primários quantitativos ou qualitativos, sem limite temporal. Selecionaram-se 24 publicações entre $2009 e$ 2020, que foram subdivididas de acordo com as abordagens da avaliação: avaliabilidade, sustentabilidade, processo (oferta e estrutura), resultado (impacto e satisfação) e grau de inferência (adequação, plausibilidade e probabilidade). Os resultados das avaliações mostraram que os programas oferecem diversas atividades, impactam positivamente nos indicadores de saúde dos usuários e contribuem para o aumento de atividade física no lazer. A avaliação desses programas é fundamental para a gestão, serviços de saúde e profissionais, pois permite verificar a implementação das ações propostas, a cobertura, o acesso $e$ o impacto, bem como a interferência do contexto politico na sua continuidade.

Avaliação de Programas e Projetos de Saúde; Promoção da Saúde; Atividade Motora; Revisão

\author{
Correspondência \\ A. G. Silva \\ Escola de Enfermagem, Universidade Federal de Minas Gerais. \\ Av. Prof. Alfredo Balena 190, Belo Horizonte, MG 30130-100, \\ Brasil. \\ alannagomessilva@gmail.com \\ 1 Escola de Enfermagem, Universidade Federal de Minas Gerais, \\ Belo Horizonte, Brasil.
}




\section{Introdução}

A prática insuficiente de atividade física constitui um dos principais fatores de risco modificável para as doenças crônicas não transmissíveis (DCNT), reduz a expectativa de vida e afeta negativamente a saúde mental e a qualidade de vida 1. Em 2016, 27,5\% da população adulta no mundo eram insuficientemente ativos 1. No Brasil, em 2013, o percentual foi de 46\% 2. Esse cenário desfavorável estimulou organizações internacionais e nacionais a incluírem a atividade física na agenda global de saúde 3 .

No contexto nacional, políticas, programas e ações foram implementados com o objetivo de incentivar e aumentar a prática de atividade física 4. Destaca-se o Programa Academia da Cidade (PAC), criado como uma estratégia local a partir de 2002, inicialmente em Recife (Pernambuco), e nos anos seguintes, foi implantado em outros municípios do Brasil. O PAC tem como objetivos promover hábitos de vida saudáveis, aumentar o nível de atividade física da população e ampliar o conhecimento sobre os benefícios da sua prática 3,5. Em 2006, foi aprovada a Política Nacional de Promoção da Saúde (PNPS) que instituiu a atividade física como eixo prioritário na agenda nacional 6 . A PNPS também tornou possível a criação de um programa comunitário de incentivo à prática de atividade física, no âmbito do Sistema Único de Saúde (SUS), o Programa Academia da Saúde (PAS), instituído pelo Ministério da Saúde em 2011 4,7,8.

O PAS tem como principal objetivo a promoção da saúde da população a partir da implantação de polos em todo o Brasil, com infraestrutura, equipamentos e quadro de profissional diversificado 9 . O programa complementa e potencializa as ações da atenção primária à saúde (APS) e tem referência territorial, sendo um ponto de atenção nas redes de saúde 10. O PAS também possibilitou a incorporação e a expansão de programas comunitários de atividade física, além de repasses financeiros àqueles que aderissem ao programa 11,12. Entre os programas municipais preexistentes que aderiram ao PAS destacam-se os PACs de Aracaju (Sergipe), Belo Horizonte (Minas Gerais) e Recife. A incorporação desses programas, habilitados como polos similares, permitiu a horizontalidade no processo de implementação e a continuidade de ações de promoção de atividade física no Brasil 4,13. O PAC e o PAS ampliaram o acesso e oportunizaram a prática de atividade física para a população, inclusive para os mais vulneráveis. Por isso, compreende-se que esses programas promovem a saúde e materializam os princípios do SUS de universalidade, equidade e integralidade 14.

A vigilância, monitoramento e avaliação constituem eixos operacionais da PNPS 6 . A avaliação oferece ferramentas de aprimoramento, gestão e fortalecimento da APS, especialmente em tempos de instabilidade político-econômica e ameaça a direitos sociais e de saúde 7,13 . Por isso, a avaliação deve ser um objeto contínuo e permanente, com vistas a reorientar as ações e subsidiar decisões, intervenções e a implantação de políticas públicas de saúde 7,13. O Ministério da Saúde investiu na avaliação dos programas comunitários de atividade física e consolidou parcerias nacionais e internacionais que favoreceram a construção de importantes evidências sobre os programas 15 . No entanto, a avaliação de programas de promoção da saúde é um processo desafiador devido à multiplicidade de ações, multidisciplinaridade, heterogeneidade dos problemas locais e regionais, e a diversidade cultural e socioeconômica 16. Por isso, as avaliações devem ser desenvolvidas com um conjunto de saberes e práticas com influência de distintas abordagens, disciplinas científicas e tradições teórico-metodológicas 17 , que sejam capazes de revelar evidências relevantes para o programa 18. No entanto, não foram encontrados estudos que mapeassem as evidências sobre as abordagens utilizadas, bem como os resultados obtidos das avaliações do PAC e PAS que permitissem criar generalidade sobre os mecanismos e estratégias desenvolvidos por estes programas para a promoção da atividade física no Brasil.

Nesse sentido, os objetivos neste estudo foram identificar e sintetizar as evidências científicas sobre as abordagens e resultados das avaliações realizadas no PAS e no PAC.

\section{Métodos}

Trata-se de uma revisão de escopo que consiste em sintetizar as evidências de pesquisas, para mapear a literatura existente de determinado assunto em termos de natureza, características e volume ${ }^{19}$. Esta revisão teve o protocolo de pesquisa registrado no Open Science Framework no dia 27 de julho de 2020 (https://osf.io/zk6eg/). Foi desenvolvida com base nas recomendações do guia internacional Preferred 
Reporting Items for Systematic reviews and Meta-Analyses extension for Scoping Reviews (PRISMA-ScR) $20 \mathrm{e}$ pelo método proposto pelo Instituto Joanna Briggs (JBI) 21.

Para orientar a formulação da questão norteadora, adotou-se a estratégia População, Conceito e Contexto (PCC) com a seguinte pergunta: quais as evidências científicas sobre as abordagens e resultados das avaliações realizadas no PAS e no PAC? Assim, foram definidos com base na questão norteadora: População - usuários, profissionais de saúde e gestores envolvidos nas avaliações do programa; Conceito - abordagens e resultados das avaliações; e Contexto - PAS e PAC.

\section{Critério de elegibilidade dos estudos}

Foram incluídos na revisão estudos primários quantitativos ou qualitativos. Consideraram-se também teses, dissertações, livros, documentos técnicos e governamentais, e não houve limite temporal para a seleção. Incluíram-se publicações em inglês, espanhol e português que continham os seguintes descritores ou palavras-chave: estudos de avaliação, avaliação de programas e projetos de saúde, avaliação de processos e resultados em cuidados de saúde, academia(s) da saúde, e academia(s) da cidade. Excluíram-se aqueles que não tiveram como objetivo principal a avaliação do PAS ou PAC, que não abordaram especificamente estes programas, bem como revisões narrativas e integrativas, textos da Internet, editoriais, ensaios e artigos não disponibilizados na íntegra nas bases de dados.

\section{Fontes de informação e estratégia de busca}

Realizaram-se as buscas entre março e junho de 2020. A busca da produção científica foi feita nas bases de dados MEDLINE (via PubMed), LILACS (via Biblioteca Virtual em Saúde), Scopus (via Portal CAPES) e Cochrane (via Portal CAPES), no site do PAS/Ministério da Saúde, no Catálogo de Teses e Dissertações da Coordenação de Aperfeiçoamento de Pessoal de Nível Superior (CAPES) e na Biblioteca Digital Brasileira de Teses e Dissertações (BDTD) do Instituto Brasileiro de Informação em Ciência e Tecnologia (IBICT).

As referências dos artigos selecionados foram verificadas para identificar novos estudos não localizados nas buscas anteriores, observados os critérios de inclusão previamente estabelecidos.

Considerando os critérios de inclusão, elaborou-se a estratégia de busca no PubMed, a partir do Medical Subject Headings (MeSH) e acrescidos das palavras-chave a saber: "Academia da Saúde" OR "Academias da Saúde" OR "Academia da Cidade" OR "Academias da Cidade" OR "Health Academy" OR "City Academy" AND "Evaluation Study" [MeSH Terms] OR "Program Evaluations" [MeSH Terms] OR "Outcome and Process Assessment, Health Care" [MeSH Terms] OR "Evaluation Study" OR "Program Evaluations" OR "Outcome and Process Assessment, Health Care".

Essa estratégia foi adaptada conforme as especificidades de cada base utilizada. Em todas as bases de dados realizou-se a busca considerando-se a data de publicação até o dia 4 de maio de 2020.

Os resultados da pesquisa final foram exportados para o Mendeley (https://www.mendeley.com) e os duplicados foram removidos.

\section{Seleção de fontes de evidência}

Dois revisores fizeram a triagem independente dos estudos e os selecionaram com base nos títulos e resumo. Posteriormente, os revisores leram independentemente na íntegra os artigos pré-selecionados, identificando com precisão a sua relevância para a pesquisa e se os critérios de inclusão estavam contemplados. As divergências entre os revisores foram resolvidas por discussão e em colaboração com um terceiro revisor, para alcançar o consenso entre todos.

\section{Processo de coleta dos dados e síntese dos resultados}

A extração e sintetização dos elementos essenciais encontrados em cada publicação foram realizadas por dois revisores independentes, a partir de um instrumento estruturado, elaborado para este estudo, e utilizou-se o Microsoft Excel (https://products.office.com/) para a tabulação dos dados. 
Os dados extraídos incluíram detalhes sobre a autoria, ano das publicações, tipo (artigo, dissertação e documentos governamentais), objetivos, desenho, local, níveis de evidência, programa avaliado, população, abordagens das avaliações (tipo de avaliação, indicadores e grau de inferência) e as principais descobertas relevantes para o objetivo desta revisão.

O nível de evidência e o grau de recomendação dos estudos foram categorizados conforme a classificação do JBI 22 e estão apresentados no Quadro 1.

Categorizaram-se as abordagens avaliativas quanto ao tipo de avaliação, indicadores e, quando aplicável, ao grau de inferência. Como os autores usaram diversas abordagens avaliativas, as categorias foram fundamentadas nos marcos conceituais de avaliação, segundo Vieira-da-Silva 23, Pluye et al. 24, Habicht et al. 25 e Donabedian 26 (Quadro 2).

A síntese dos achados foi feita pela similaridade das temáticas e usou-se a estatística descritiva para a análise dos resultados, por meio de frequência absoluta e relativa.

\section{Resultados}

Identificaram-se, pela estratégia de busca, 255 artigos e outros 217 foram incluídos de outras fontes ( $\mathrm{n}$ = 472). Excluíram-se 20 documentos duplicados e 317 que não atendiam aos critérios de inclusão pela leitura do título. Selecionaram-se para leitura do resumo 135 estudos e, posteriormente, 89 foram excluídos, por não cumprirem os critérios de inclusão. Por fim, 46 foram lidos na íntegra e, destes, 22 foram excluídos pelos seguintes motivos: não tinham como objetivo a avaliação dos programas (16/22), artigos de revisão (2/22) e artigos que não abordaram o PAC ou o PAS (4/22). Ao final, obtevese o total de 24 estudos incluídos nesta revisão (Figura 1).

\section{Quadro 1}

Nível de evidência e grau de recomendação dos estudos, segundo a classificação do Instituto Joanna Briggs 22.

\begin{tabular}{|c|c|}
\hline NÍVEL DE EVIDÊNCIA & GRAU DE RECOMENDAÇÃO \\
\hline \multirow[t]{4}{*}{ Nível 1: Estudos experimentais } & 1.a - Revisão sistemática de ensaios clínicos randomizados controlados. \\
\hline & 1.b - Revisão sistemática de ensaios clínicos randomizados controlados e outros desenhos de estudo. \\
\hline & 1.c - Ensaio clínico randomizado controlado. \\
\hline & 1.d - Pseudo ensaio clínico randomizado controlado. \\
\hline \multirow{4}{*}{$\begin{array}{l}\text { Nível 2: Estudos quase } \\
\text { experimentais }\end{array}$} & 2.a - Revisão sistemática de estudos quase experimentais. \\
\hline & 2.b - Revisão sistemática de estudos quase experimentais e outros desenhos de menor evidência. \\
\hline & 2.c - Estudo prospectivo controlado quase experimental. \\
\hline & 2.d - Pré-teste e pós-teste ou estudo de grupo controle histórico/retrospectivo. \\
\hline \multirow{5}{*}{$\begin{array}{l}\text { Nível 3: Estudos analíticos } \\
\text { observacionais }\end{array}$} & 3.a - Revisão sistemática de estudos de coortes comparáveis. \\
\hline & 3.b - Revisão sistemática de coortes comparáveis e outros desenhos de estudo de menor evidência. \\
\hline & 3.c - Estudo de coorte com grupo controle. \\
\hline & 3.d - Estudo caso controle. \\
\hline & 3.e - Estudos observacionais sem um grupo controle. \\
\hline \multirow{4}{*}{$\begin{array}{l}\text { Nível 4: Estudos descritivos } \\
\text { observacionais }\end{array}$} & 4.a - Revisão sistemática de estudos descritivos. \\
\hline & 4.b - Estudo transversal. \\
\hline & 4.c - Séries de casos. \\
\hline & 4.d - Estudo de caso. \\
\hline \multirow{3}{*}{$\begin{array}{l}\text { Nível 5: Opinião de especialista e } \\
\text { pesquisas de bancada }\end{array}$} & 5.a - Revisão sistemática de opinião de especialistas. \\
\hline & 5.b-Consenso de especialistas. \\
\hline & 5.c - Pesquisa de bancada/opinião de um especialista. \\
\hline
\end{tabular}


Quadro 2

Categorização das abordagens avaliativas dos estudos, segundo tipo de avaliação.

\begin{tabular}{|c|c|}
\hline \multicolumn{2}{|r|}{ TIPOS DE AVALIAÇÃO } \\
\hline Avaliabilidade & $\begin{array}{l}\text { Análise sistemática e preliminar de um programa em sua teoria e prática para determinar se há } \\
\text { justificativa para uma avaliação } 23 \text {. }\end{array}$ \\
\hline Sustentabilidade & $\begin{array}{l}\text { Refere-se à probabilidade de um programa ser capaz de continuar suas atividades operacionais e } \\
\qquad \text { manter seus resultados ao longo do tempo } 24 \text {. }\end{array}$ \\
\hline Processo (oferta e cobertura) & $\begin{array}{l}\text { Tem o objetivo de produzir conhecimento para uso local sobre o que está sendo oferecido à população. } \\
\text { O indicador de oferta visa a responder se as ações ou atividades do programa estão disponíveis para } \\
\text { a população-alvo, se são acessíveis e de qualidade. A cobertura avalia a proporção da população-alvo } \\
\text { atingida pelo programa } 25 .\end{array}$ \\
\hline Resultado (impacto e satisfação) & $\begin{array}{l}\text { Compreende os efeitos de um cuidado sobre a saúde dos indivíduos ou populações } 26 \text {. A satisfação do } \\
\text { usuário ou do profissional também é outra dimensão do resultado, estando relacionada à qualidade e } \\
\text { à eficácia do cuidado } 26, \text { e o indicador de impacto avalia se os objetivos do programa foram alcançados } \\
\text { ou não } 25 .\end{array}$ \\
\hline \multicolumn{2}{|r|}{ GRAU DE INFERÊNCIA * } \\
\hline Adequação & Avalia se as mudanças esperadas aconteceram ou não 25. \\
\hline Plausibilidade & $\begin{array}{l}\text { Vai além da adequação, pois investiga se o efeito observado ocorreu devido ao programa e tenta } \\
\text { controlar o efeito de fatores de confusão, utilizando grupos-controle, mas sem randomização } 25 \text {. }\end{array}$ \\
\hline Probabilidade & $\begin{array}{l}\text { Estima a probabilidade estatística de que o programa tenha realmente efeito, requer randomização dos } \\
\qquad \text { grupos, sendo o padrão-ouro das pesquisas de eficácia } 25 \text {. }\end{array}$ \\
\hline
\end{tabular}

* Refere-se ao tipo de inferência necessária (adequação, plausibilidade e probabilidade) para se afirmar que os resultados observados, tanto de processo quanto de impacto, foram efetivamente decorrentes da intervenção 25.

\section{Características das publicações}

Dos 24 estudos selecionados, a maioria foi de artigos $(\mathrm{n}=20)$, seguidos de documentos governamentais $(\mathrm{n}=2)$, capítulo de livro $(\mathrm{n}=1)$ e dissertação $(\mathrm{n}=1)$, os quais são transversais $(\mathrm{n}=14)$, qualitativos $(\mathrm{n}=8)$, quase experimental $(\mathrm{n}=1)$ e quali-quantitativo $(\mathrm{n}=1)$. Foram desenvolvidos em Recife $(\mathrm{n}=9)$, Belo Horizonte $(n=5)$, no Brasil $(n=4)$, no conjunto das capitais brasileiras $(n=1)$, em Aracaju $(n=1)$, Cariri ( $\mathrm{n}=1)$, Florianópolis $(\mathrm{n}=1)$, Rio de Janeiro $(\mathrm{n}=1)$ e Pernambuco $(\mathrm{n}=1)$, sendo publicados entre 2009 e 2020. Os níveis de evidência foram categorizados em 4.b - Estudo transversal ( $\mathrm{n}=15)$, 4.d Estudo de caso $(\mathrm{n}=8)$ e 2.c - Estudo prospectivo controlado quase experimental ( $=1)$ (Quadro 3).

\section{Descrição, contexto e características das avaliações}

As avaliações foram realizadas no PAC $(\mathrm{n}=12)$, PAS $(\mathrm{n}=11)$ e em ambos os programas $(\mathrm{n}=1)$. Participaram das avaliações usuários, ex-usuários e não usuários dos programas, gestores, coordenadores, profissionais de saúde e responsáveis pelo programa nos municípios. Realizaram-se pesquisas sobre avaliabilidade, avaliações de sustentabilidade, de processo (oferta e cobertura), resultado (impacto e satisfação) e grau de inferência (adequação, plausibilidade e probabilidade) (Quadro 4).

Os principais resultados dos estudos foram sumarizados de acordo com as abordagens da avaliação: avaliabilidade, sustentabilidade, processo (oferta e estrutura), resultado (impacto e satisfação) e grau de inferência (adequação, plausibilidade e probabilidade). 


\section{Figura 1}

Fluxograma PRISMA de seleção de estudos 60.
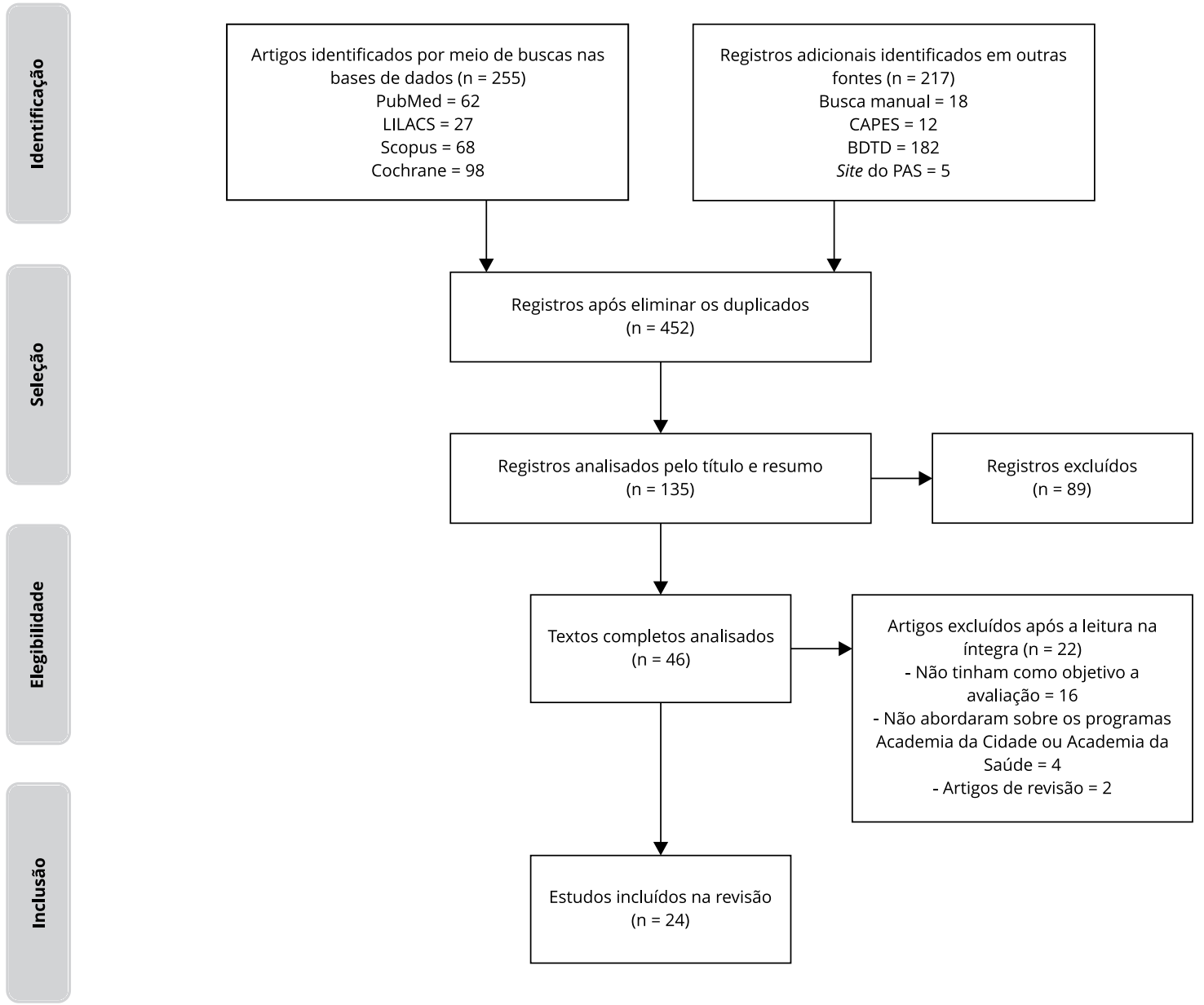

BDTD: Biblioteca Digital Brasileira de Teses e Dissertações; CAPES: Coordenação de Aperfeiçoamento de Pessoal de Nível Superior;

PAS: Programa Academia da Saúde.

\section{- Avaliabilidade}

A avaliabilidade do programa foi encontrada em dois estudos, um realizado no PAC no Rio de Janeiro 27 e outro no PAS em Recife 28.

Os resultados da avaliabilidade do Programa Academia Carioca da Saúde permitiram evidenciar a adequação do desenho do programa, pois são implementadas as diretrizes da PNPS 27. Em contrapartida, a avaliabilidade do PAS de Recife demonstrou que as diretrizes do programa são muito abrangentes e pouco esclarecedoras quanto ao planejamento, operacionalização e avaliação das ações. Verificou-se, ainda, que parte dos gestores desconhece os objetivos, princípios e diretrizes do programa 28 .

A construção do modelo lógico também foi realizada em ambos os estudos, sendo este um instrumento de apoio ao planejamento e avaliação do programa ao sistematizar os recursos existentes, atividades, contexto e resultados esperados 27,28. O modelo lógico permitiu descrever as dimensões e os componentes do programa, as ações estratégicas e os efeitos esperados com a implantação do 
Quadro 3

Caracterização das publicações segundo autores, ano, tipo, objetivos, desenho do estudo, local e nível de evidência.

\begin{tabular}{|c|c|c|c|c|c|}
\hline $\begin{array}{l}\text { AUTORES } \\
\text { (ANO) }\end{array}$ & TIPO & OBJETIVO & $\begin{array}{l}\text { DESENHO DO } \\
\text { ESTUDO }\end{array}$ & LOCAL & $\begin{array}{l}\text { NÍVEL DE } \\
\text { EVIDÊNCIA * }\end{array}$ \\
\hline $\begin{array}{l}\text { Hallal et al. } 36 \\
(2009)\end{array}$ & Artigo & $\begin{array}{c}\text { Identificar e analisar a visão que os professores } \\
\text { constroem sobre o impacto, relevância, dificuldades e } \\
\text { envolvimento da comunidade }\end{array}$ & $\begin{array}{l}\text { Quali- } \\
\text { quantitativo }\end{array}$ & $\begin{array}{c}\text { Recife } \\
\text { (Pernambuco) }\end{array}$ & 4.b \\
\hline $\begin{array}{l}\text { Simões et al. } 37 \\
(2009)\end{array}$ & Artigo & $\begin{array}{l}\text { Avaliar os efeitos do PAC sobre o aumento da atividade } \\
\text { física no lazer }\end{array}$ & Transversal & $\begin{array}{c}\text { Recife } \\
\text { (Pernambuco) }\end{array}$ & 4.b \\
\hline $\begin{array}{l}\text { Hallal et al. } 3 \\
(2010)\end{array}$ & Artigo & Descrever o perfil dos usuários e não usuários do PAC & Transversal & $\begin{array}{c}\text { Recife } \\
\text { (Pernambuco) }\end{array}$ & 4.b \\
\hline $\begin{array}{l}\text { Mendonça et al. } \\
38 \text { (2010) }\end{array}$ & Artigo & $\begin{array}{c}\text { Avaliar a associação entre várias formas de exposição } \\
\text { ao PAC e atividade física no lazer }\end{array}$ & Transversal & $\begin{array}{l}\text { Aracaju } \\
\text { (Sergipe) }\end{array}$ & $4 . b$ \\
\hline $\begin{array}{l}\text { Mazo et al. } 31 \\
(2013)\end{array}$ & Artigo & $\begin{array}{c}\text { Verificar a percepção dos participantes quanto aos } \\
\text { serviços prestados, aos ingressos e permanência, e ao } \\
\text { nível de atividade física }\end{array}$ & Transversal & $\begin{array}{c}\text { Florianópolis } \\
\text { (Santa Catarina) }\end{array}$ & 4.b \\
\hline $\begin{array}{l}\text { Silva et al. } 32 \\
(2014)\end{array}$ & Artigo & $\begin{array}{c}\text { Analisar o PAC sob a perspectiva de } \\
\text { usuários e monitores }\end{array}$ & Qualitativo & $\begin{array}{l}\text { Belo Horizonte } \\
\text { (Minas Gerais) }\end{array}$ & 4.d \\
\hline $\begin{array}{l}\text { Ministério da } \\
\text { Saúde } 33 \text { (2015) }\end{array}$ & $\begin{array}{c}\text { Documento } \\
\text { governamental }\end{array}$ & Monitorar o PAS nos municípios brasileiros & Transversal & Brasil & 4.b \\
\hline $\begin{array}{l}\text { Fernandes } \\
\text { et al. } 39 \text { (2015) }\end{array}$ & Artigo & $\begin{array}{l}\text { Avaliar o efeito do PAC sobre a prática de atividade } \\
\text { física no lazer de não usuários em domicílios } \\
\text { localizados a diferentes distâncias da academia }\end{array}$ & Transversal & $\begin{array}{l}\text { Belo Horizonte } \\
\text { (Minas Gerais) }\end{array}$ & 4.b \\
\hline $\begin{array}{l}\text { Padilha et al. } 27 \\
\text { (2015) }\end{array}$ & Artigo & $\begin{array}{l}\text { Apresentar os resultados do estudo de avaliabilidade } \\
\text { do Programa Academia Carioca da Saúde }\end{array}$ & Qualitativo & Rio de Janeiro & 4.d \\
\hline $\begin{array}{l}\text { Paez et al. } 29 \\
(2015)\end{array}$ & Artigo & $\begin{array}{l}\text { Avaliar e relatar os elementos de validade } \\
\text { externa do PAC }\end{array}$ & Qualitativo & $\begin{array}{c}\text { Recife } \\
\text { (Pernambuco) }\end{array}$ & 4.d \\
\hline $\begin{array}{l}\text { Feitosa et al. } 40 \\
\text { (2016) }\end{array}$ & Artigo & $\begin{array}{l}\text { Analisar a percepção das usuárias do PAC do Recife } \\
\text { quanto a satisfação com a oferta de serviços e } \\
\text { percepção de mudanças ligadas à qualidade de vida } \\
\text { após a entrada no programa }\end{array}$ & Qualitativo & $\begin{array}{c}\text { Recife } \\
\text { (Pernambuco) }\end{array}$ & 4.d \\
\hline $\begin{array}{l}\text { Florindo et al. } 34 \\
(2016)\end{array}$ & Artigo & $\begin{array}{c}\text { Avaliar a promoção da atividade física e alimentação } \\
\text { saudável em municípios que receberam recursos para } \\
\text { o desenvolvimento do PAS }\end{array}$ & Transversal & Brasil & 4.b \\
\hline Sá et al. ${ }^{4}$ (2016) & Artigo & $\begin{array}{l}\text { Descrever o cenário da implantação do PAS e as } \\
\text { características de funcionamento }\end{array}$ & Transversal & Brasil & 4.b \\
\hline $\begin{array}{l}\text { Fernandes } \\
\text { et al. }{ }^{13}(2017)\end{array}$ & Artigo & $\begin{array}{c}\text { Descrever o histórico e a metodologia de } \\
\text { avaliação do PAS }\end{array}$ & Transversal & $\begin{array}{l}\text { Belo Horizonte } \\
\text { (Minas Gerais) }\end{array}$ & 4.b \\
\hline $\begin{array}{l}\text { Silva et al. } 28 \\
(2017)\end{array}$ & Artigo & Realizar um estudo da avaliabilidade do PAS & Qualitativo & $\begin{array}{c}\text { Recife } \\
\text { (Pernambuco) }\end{array}$ & 4.d \\
\hline $\begin{array}{l}\text { Simões et al. } 41 \\
(2017)\end{array}$ & Artigo & Avaliar o impacto do PAC na prática de atividade física & $\begin{array}{c}\text { Quase } \\
\text { experimental }\end{array}$ & $\begin{array}{c}\text { Recife } \\
\text { (Pernambuco) }\end{array}$ & 2.c \\
\hline $\begin{array}{l}\text { Andrade et al. } 42 \\
(2018)\end{array}$ & Artigo & $\begin{array}{l}\text { Avaliar o efeito do PAC na prática de atividade física no } \\
\text { lazer por não usuários residentes nas proximidades } \\
\text { dos polos }\end{array}$ & Transversal & $\begin{array}{l}\text { Belo Horizonte } \\
\text { (Minas Gerais) }\end{array}$ & 4.b \\
\hline $\begin{array}{l}\text { Ministério da } \\
\text { Saúde } 10 \text { (2018) }\end{array}$ & $\begin{array}{c}\text { Documento } \\
\text { governamental }\end{array}$ & Monitorar o PAS nos municípios brasileiros & Transversal & Brasil & 4.b \\
\hline $\begin{array}{l}\text { Cazarin et al. } 30 \\
(2019)\end{array}$ & Artigo & Analisar a sustentabilidade do PAC & Qualitativo & $\begin{array}{c}\text { Recife } \\
\text { (Pernambuco) }\end{array}$ & 4.d \\
\hline
\end{tabular}

(continua) 
Quadro 3 (continuação)

\begin{tabular}{|c|c|c|c|c|c|}
\hline $\begin{array}{l}\text { AUTORES } \\
\text { (ANO) }\end{array}$ & TIPO & OBJETIVO & $\begin{array}{l}\text { DESENHO DO } \\
\text { ESTUDO }\end{array}$ & LOCAL & $\begin{array}{l}\text { NÍVEL DE } \\
\text { EVIDÊNCIA * }\end{array}$ \\
\hline $\begin{array}{l}\text { Gonçalves } \\
\text { et al. } 43 \text { (2019) }\end{array}$ & Artigo & $\begin{array}{l}\text { Analisar a percepção sobre a operacionalidade, as } \\
\text { ações e a integração com a rede de saúde }\end{array}$ & Qualitativo & Cariri (Ceará) & 4.d \\
\hline $\begin{array}{l}\text { Maciel et al. } 44 \\
\text { (2019) }\end{array}$ & Artigo & $\begin{array}{l}\text { Adotar os parâmetros de desempenho para avaliar um } \\
\text { polo do PAS }\end{array}$ & Qualitativo & $\begin{array}{l}\text { Belo Horizonte } \\
\text { (Minas Gerais) }\end{array}$ & 4.d \\
\hline Melo 45 (2019) & $\begin{array}{l}\text { Dissertação de } \\
\text { Mestrado }\end{array}$ & Avaliar o PAC considerando dois critérios de avaliação & Transversal & $\begin{array}{c}\text { Recife } \\
\text { (Pernambuco) }\end{array}$ & 4.b \\
\hline $\begin{array}{l}\text { Faria et al. } 46 \\
(2020)\end{array}$ & $\begin{array}{l}\text { Capítulo de } \\
\text { livro }\end{array}$ & $\begin{array}{c}\text { Analisar o impacto do PAS na prática de atividade física } \\
\text { no lazer e a ingestão de frutas, legumes e hortaliças }\end{array}$ & Transversal & $\begin{array}{c}\text { Capitais } \\
\text { brasileiras }\end{array}$ & 4.b \\
\hline $\begin{array}{l}\text { Silva et al. } 35 \\
(2020)\end{array}$ & Artigo & $\begin{array}{c}\text { Avaliar as barreiras e os facilitadores para a } \\
\text { participação no PAC e no PAS }\end{array}$ & Transversal & Pernambuco & 4.b \\
\hline
\end{tabular}

PAC: Programa Academia da Cidade; PAS: Programa Academia da Saúde.

* Consultar Quadro 1.

\section{Quadro 4}

Características das avaliações, segundo o nome do programa, a população, tipos de avalições, indicadores e grau de inferência.

\begin{tabular}{|c|c|c|c|c|}
\hline $\begin{array}{l}\text { AUTORES } \\
\text { (ANO) }\end{array}$ & $\begin{array}{c}\text { NOME DO PROGRAMA } \\
\text { AVALIADO }\end{array}$ & POPULAÇÃO & $\begin{array}{l}\text { TIPOS DE AVALIAÇÕES } \\
\text { (INDICADORES) }\end{array}$ & $\begin{array}{c}\text { GRAU DE } \\
\text { INFERÊNCIA }\end{array}$ \\
\hline $\begin{array}{l}\text { Hallal et al. } 36 \\
(2009)\end{array}$ & PAC & Profissionais de educação física & Resultado (impacto e satisfação) & Adequação \\
\hline $\begin{array}{l}\text { Simões et al. } 37 \\
(2009)\end{array}$ & PAC & Usuários e não usuários & Resultado (impacto) & Plausibilidade \\
\hline $\begin{array}{l}\text { Hallal et al. } 3 \\
(2010)\end{array}$ & PAC & Usuários e não usuários & $\begin{array}{l}\text { Processo (oferta) e resultado } \\
\text { (impacto e satisfação) }\end{array}$ & Adequação \\
\hline $\begin{array}{l}\text { Mendonça et } \\
\text { al. } 38 \text { (2010) }\end{array}$ & PAC & Usuários & Resultado (impacto) & Plausibilidade \\
\hline $\begin{array}{l}\text { Mazo et al. } 31 \\
(2013)\end{array}$ & PAS & Usuários & $\begin{array}{l}\text { Processo (oferta) e resultado } \\
\text { (satisfação) }\end{array}$ & Adequação \\
\hline $\begin{array}{l}\text { Silva et al. } 32 \\
(2014)\end{array}$ & PAC & Profissionais de educação física e usuários & $\begin{array}{l}\text { Processo (oferta) e resultado } \\
\quad \text { (impacto e satisfação) }\end{array}$ & Adequação \\
\hline $\begin{array}{l}\text { Ministério da } \\
\text { Saúde } 33 \text { (2015) }\end{array}$ & PAS & Gestores & Processo (oferta e cobertura) & Adequação \\
\hline $\begin{array}{l}\text { Fernandes et } \\
\text { al. } 39(2015)\end{array}$ & PAC & Não usuários & Resultado (impacto) & Plausibilidade \\
\hline $\begin{array}{l}\text { Padilha et al. } 27 \\
\text { (2015) }\end{array}$ & PAS & $\begin{array}{c}\text { Profissionais de educação física, gestores e } \\
\text { outros profissionais de saúde }\end{array}$ & Avaliabilidade & - \\
\hline $\begin{array}{l}\text { Paez et al. } 29 \\
(2015)\end{array}$ & PAC & Gestores e profissionais de educação física & $\begin{array}{l}\text { Processo (oferta e cobertura) e } \\
\text { sustentabilidade }\end{array}$ & Adequação \\
\hline $\begin{array}{l}\text { Feitosa et al. } 40 \\
(2016)\end{array}$ & PAS & Usuários & Resultado (impacto e satisfação) & Adequação \\
\hline
\end{tabular}

(continua) 


\begin{tabular}{|c|c|c|c|c|}
\hline $\begin{array}{l}\text { AUTORES } \\
\text { (ANO) }\end{array}$ & $\begin{array}{l}\text { NOME DO PROGRAMA } \\
\text { AVALIADO }\end{array}$ & POPULAÇÃO & $\begin{array}{l}\text { TIPOS DE AVALIAÇÕES } \\
\text { (INDICADORES) }\end{array}$ & $\begin{array}{c}\text { GRAU DE } \\
\text { INFERÊNCIA }\end{array}$ \\
\hline $\begin{array}{l}\text { Florindo } \\
\text { et al. } 34 \text { (2016) }\end{array}$ & PAS & Gestores & Processo (oferta) & Adequação \\
\hline Sá et al. 4 (2016) & PAS & Responsáveis pelo programa & Processo (oferta e cobertura) & Adequação \\
\hline $\begin{array}{l}\text { Fernandes et } \\
\text { al. } 13 \text { (2017) }\end{array}$ & PAS & Usuários e não usuários & Resultado (impacto) & Plausibilidade \\
\hline $\begin{array}{l}\text { Silva et al. } 28 \\
(2017)\end{array}$ & PAC & $\begin{array}{l}\text { Gestores, coordenadores e professores do } \\
\text { programa }\end{array}$ & Avaliabilidade & - \\
\hline $\begin{array}{l}\text { Simões et al. } 41 \\
\text { (2017) }\end{array}$ & PAC & Usuários e não usuários & Resultado (impacto) & Probabilidade \\
\hline $\begin{array}{l}\text { Andrade et al. } \\
42(2018)\end{array}$ & PAC & Não usuários & Resultado (impacto) & Plausibilidade \\
\hline $\begin{array}{l}\text { Ministério da } \\
\text { Saúde } 10 \text { (2018) }\end{array}$ & PAS & Gestores & Processo (oferta e cobertura) & Adequação \\
\hline $\begin{array}{l}\text { Cazarin et al. } 30 \\
(2019)\end{array}$ & PAC & Profissionais de saúde, usuários e gestores & Sustentabilidade & - \\
\hline $\begin{array}{l}\text { Gonçalves et al. } \\
43 \text { (2019) }\end{array}$ & PAS & Profissionais de saúde, usuários e gestores & Resultado (impacto e satisfação) & Adequação \\
\hline $\begin{array}{l}\text { Maciel et al. } 44 \\
\text { (2019) }\end{array}$ & PAS & $\begin{array}{l}\text { Profissionais de educação física, usuários e } \\
\text { ex-alunos }\end{array}$ & Resultado (impacto) & Adequação \\
\hline Melo 45 (2019) & PAC & Usuários & Resultado (impacto) & Adequação \\
\hline $\begin{array}{l}\text { Faria et al. } 46 \\
(2020)\end{array}$ & PAS & Usuários e não usuários & Resultado (impacto) & Plausibilidade \\
\hline $\begin{array}{l}\text { Silva et al. } 35 \\
(2020)\end{array}$ & PAC e PAS & Usuários & Processo (oferta) & Adequação \\
\hline
\end{tabular}

PAC: Programa Academia da Cidade; PAS: Programa Academia da Saúde.

programa, bem como identificar os recursos, atividades e as perguntas avaliativas 27,28. Os resultados também mostraram a viabilidade da realização de avaliações dos programas 27,28.

\section{- Avaliação de sustentabilidade}

A sustentabilidade dos programas também foi evidenciada por dois trabalhos 29,30, os quais revelaram fatores favoráveis e limites à sustentabilidade.

(i) Fatores favoráveis: programa como espaço de formação multiprofissional; articulação intra e intersetorial; fortalecimento da participação social na gestão do PAC; reconhecimento nacional e internacional; cofinanciamento estadual e federal 30; comprometimento do setor público; alocação de recursos; gerenciamento e estrutura do programa; qualidade da equipe; reconhecimento da atividade física como prioridade de saúde pelo Ministério da Saúde ${ }^{29}$.

(ii) Limites: falta de pessoal, de financiamento 29 e redução dos investimentos 30.

\section{- Avaliação de processo}

O processo do PAS e do PAC foi avaliado em nove estudos. Destes, todos utilizaram o indicador de oferta 3,4,10,29,31,32,33,34,35 e quatro também verificam a cobertura 4,10,29,33.

Para a participação nos programas não há critérios de inclusão ou exclusão ${ }^{29}$. A maior parte da população que participava das atividades dos programas era de adultos, seguidos de idosos, adoles- 
centes e crianças 4,10,33. Em relação à população em situação de vulnerabilidade, os negros e as pessoas com deficiência foram os grupos com a maior participação nas atividades desenvolvidas nos polos 10 . No entanto, houve baixo percentual da participação de migrantes, quilombolas, ribeirinhos, indígenas, pessoas em situação de rua e ciganos 4,10,33.

Até maio de 2017 havia 3.821 polos do PAS habilitados em todos os estados brasileiros, em diferentes etapas de implantação, e 450 polos similares 4,10,33. As atividades eram oferecidas nos turnos da manhã, tarde e noite 4,10,29,32,33, de segunda a sexta-feira 29,32 e em alguns também aos sábados 32 . A duração das sessões de atividades variou de 50 a 70 minutos 3,32, com média de 30 pessoas por sessão 32 .

As atividades oferecidas nos polos foram: práticas corporais e atividades físicas (100\%) 3,4,10,29,31,32,33,34,35; educação em saúde (44,4\%) 4,10,29,33,34; práticas integrativas e complementares em saúde (44,4\%) 4,10,33,35; avaliação física $(22,2 \%)$ 29,32; passeios e viagens $(11,1 \%) 29$.

\section{- Avaliação de resultado}

As avaliações de resultado foram abordadas por 15 estudos 3,13,31,32,36,37,38,39,40,41,42,43,44,45,46. Dois indicadores foram usados para avaliar o resultado das intervenções: o de impacto foi utilizado por 14 estudos 3,13,32,36,37,38,39,40,41,42,43,44,45,46 e o de satisfação por seis 3,31,32,36,40,43. Alguns autores utilizaram mais de um indicador, portanto, o somatório foi superior ao número total.

Das pesquisas que avaliaram o impacto $(\mathrm{n}=14)$ 3,13,32,36,37,38,39,40,41,42,43,44,45,46, 50\% ( $\mathrm{n}=7)$ utilizam como grau de inferência a adequação 3,32,36,40,43,44,45, 42,9\% (n=6) a plausibilidade 13,37,38,39,42,46 e 7,1\% ( $\mathrm{n}=1)$ a probabilidade 41 .

As avaliações de adequação mostraram que a participação no PAC ou no PAS impactou positivamente nos indicadores de saúde dos usuários 3,32,36,40,43,44,45: houve relatos de melhora da qualidade de vida 32,40,43,45, do condicionamento físico 32,44, aumento da interação social 32,40, da autoestima 43, do consumo de alimentos saudáveis 40, da redução da insônia, do estresse, do uso de medicamentos, do peso e do índice de massa corporal (IMC) 32,44, e do controle de hipertensão e diabetes 43 . Além disso, o programa impactou na redução de custos para a saúde pública e melhorou a utilização dos espaços públicos 36 .

A plausibilidade e a probabilidade mostraram resultados estatisticamente significativos: os indivíduos que participavam, que conheciam ou moravam próximos a um polo do programa tiveram mais chances de praticar atividade física no lazer ( $\geq 150$ minutos semanais) ao serem comparados com os não usuários, aqueles que não conheciam, moravam em locais distantes ou sem o polo do programa. Além disso, a prevalência dessa prática também foi maior entre os participantes e aqueles que moravam mais próximos de polos do programa 13,37,38,39,42,46.

Os usuários manifestaram satisfação com o programa 3, com o atendimento prestado 31, com as atividades desenvolvidas 40,43, com os professores do programa 40,43; com a facilidade de acesso (geográfico e econômico) 32,36,43, com a flexibilidade no horário das atividades 43 e com a infraestrutura 31 .

\section{Discussão}

Mapear a literatura a respeito das abordagens e resultados das avaliações realizadas no PAS e no PAC permitiu identificar 24 publicações entre 2009 e 2020, sendo a maioria artigos com desenho transversal $(\mathrm{n}=15)$, realizados principalmente em Recife e Belo Horizonte. As abordagens avaliativas realizadas foram referentes à avaliabilidade e sustentabilidade, ao processo e ao resultado. O PAS e o PAC apresentam pontos fortes para sustentabilidade, oferecem diversas atividades, impactam positivamente na saúde e na qualidade de vida dos usuários e contribuem para o aumento da prática de atividade física no lazer e para a melhoria dos espaços públicos.

Os 24 estudos incluídos nesta revisão evidenciaram as diversas avaliações realizadas no PAS e no PAC, tornando-se uma pesquisa abrangente por abarcar publicações dos últimos 12 anos. Esta revisão identificou estudos avaliativos publicados antes da criação do PAS (2009 e 2010) 3,36,37,38, no entanto, o maior quantitativo de pesquisas foi após 2011 4,10,13,27,28,29,30,31,32,33,34,35,40,41,42,43,44,45,46, sendo um reflexo da criação do PAS e da indução de políticas públicas no campo da promoção da saúde e da avaliação 7,11,12. Entre os marcos históricos e político-institucionais que justificam esse 
importante crescimento de estudos avaliativos, destacam-se a publicação da PNPS em 2006 e sua atualização em 2014; a criação da Rede Nacional de Promoção da Saúde, a partir de 2006; o Projeto Guia (Guia Útil para Intervenções em Atividade Física na América Latina), entre 2008 e 2011; o lançamento do Plano de Ações Estratégicas para o Enfrentamento das DCNTs, em 2011; e o próprio PAS 7,8,11. Soma-se ainda a parceria do Ministério da Saúde com universidades brasileiras e com o Centro de Controle e Prevenção de Doenças (CDC) norte-americano, que juntamente com o Projeto Guia avaliou programas e políticas de promoção de atividade física em vários estados brasileiros 11,47. Além disso, houve forte indução e financiamento do Ministério da Saúde para os estudos de avaliação do PAC e do PAS 12.

Na sequência desse processo de estímulo à avaliação do PAC e do PAS, em 2011 o Ministério da Saúde, por meio do Departamento de Ciência e Tecnologia (DECIT) e do Conselho Nacional de Desenvolvimento Científico e Tecnológico (CNPq), aprovou um projeto, em parceria com 11 universidades brasileiras, para avaliar a efetividade, implantação e implementação desses programas e as barreiras e os facilitadores para a promoção da saúde, especialmente por meio da prática de atividade física 12. Com isso, a maioria das avaliações realizadas entre 2013 e 2020 foi financiada ou induzida por esse projeto, mostrando a importância das ações de fomento para o aperfeiçoamento de políticas públicas no apoio a estudos e estímulo às avaliações 11,12,48. Destaca-se, ainda, o incentivo da Secretaria de Vigilância em Saúde do Ministério da Saúde na criação de um sistema de monitoramento do PAS, que apoiou os municípios no processo de acompanhamento da sua implantação e funcionamento no país 10 . Outro importante marco que contribuiu para o monitoramento e avaliação foi a inclusão da atividade física nos inquéritos populacionais brasileiros, como no sistema de Vigilância de Fatores de Risco e Proteção para Doenças Crônicas por Inquérito Telefônico (Vigitel), na Pesquisa Nacional de Saúde do Escolar (PeNSE) e na Pesquisa Nacional de Saúde (PNS), permitindo a vigilância da atividade física entre a população brasileira 49 .

Esta revisão identificou as seguintes abordagens avaliativas realizadas no PAC e no PAS: avaliabilidade 27,28 , sustentabilidade 29,30 , processo $3,4,10,29,31,32,33,34,35$ e resultado $3,13,31,32,36,37,38,39,40,41,42,43,44$, 45,46 , e os graus de inferência de adequação 3,4,10,29,31,32,33,34,35,36,40,43,44,45, plausibilidade 13,37,38,39,42,46 ou probabilidade ${ }^{41}$, os quais variaram de acordo com o programa, sua natureza, características e objetivos. As abordagens avaliativas estão coerentes com o previsto nos referenciais teóricos, pois devem ser selecionadas de acordo com o objeto a ser avaliado e o objetivo da avaliação, sendo que, o avaliador deve ter criatividade e conhecimento para escolher adequadamente o método que será utilizado 50 . Assim, encontram-se, primeiramente, os estudos de avaliabilidade, com a construção do modelo lógico, que correspondem ao primeiro passo para avaliar um programa, com o objetivo de determinar se o que foi planejado realmente ocorreu. Posteriormente, é necessário avaliar os produtos e o impacto do programa, verificando se os resultados, de fato, estão relacionados às ações desenvolvidas, também presente nos estudos analisados. As diversas abordagens avaliativas realizadas no PAC e no PAS mostram que a implantação da avaliação de um programa requer o desenho de uma matriz que apresente as abordagens, os critérios, indicadores e parâmetros que serão utilizados, bem como as respectivas fontes de informação 51, como apresentado nos estudos analisados.

Destaca-se que o processo avaliativo deve ser construído em bases lógicas, coerentes e racionais, usando abordagens e instrumentos de pesquisa que garantam a consistência, validade e confiabilidade dos resultados 52 . Além disso, a avaliação deve ser permanente e sistemática, articulada às ações implementadas, com vistas a subsidiar a definição de problemas, reorientar estratégias desenvolvidas, fornecer elementos para a transformação das práticas e mensurar os impactos das ações, propiciando o aumento de eficiência, eficácia e efetividade das atividades desenvolvidas ${ }^{52}$. A avaliação também favorece o aprendizado individual e coletivo, sendo um instrumento de transformação e inovação, que permite o desenvolvimento de uma cultura democrática e uma visão crítica do que foi estabelecido 53 . No entanto, ainda é necessário institucionalizar a avaliação em todos os níveis do sistema de saúde e criar uma verdadeira cultura de avaliação ${ }^{53}$, pois os serviços, as políticas públicas e os programas necessitam ser mensurados, reorientados e, sobretudo, baseados em evidências 54 .

Quanto aos níveis de evidências, o maior quantitativo foi referente ao nível 4.b - Estudo transversal 3,4,10,13,31,33,34,35,36,37,38,39,42,45,46, seguido de 4.d - Estudo de caso 27,28,29,30,32,40,43,44 e 2.c - Estudo prospectivo controlado quase experimental 41 . Em pesquisa avaliativa na área da saúde, praticamente todos os tipos de estudos epidemiológicos podem ser utilizados 55 , porém a escolha do desenho da 
investigação dependerá também do objetivo da avaliação e da disponibilidade de recursos técnicos e financeiros para a sua execução 51,55 . Em uma perspectiva metodológica quantitativa, o desenho experimental é considerado padrão-ouro para a avaliação de eficácia, contudo, é pouco aplicado, principalmente pelas questões éticas e operacionais 55 . Nas avaliações em saúde, os desenhos observacionais são mais usados, tendo em vista as questões éticas, bem como a complexidade das intervenções de saúde coletiva e sua relação com o contexto 51 , como é o caso dos estudos analisados. Os estudos de caso e o uso de técnicas qualitativas são utilizados para analisar a dimensão relacional das práticas 51 . Assim, as avaliações realizadas no PAC e no PAS mostraram diversidades conceituais, terminológicas, a utilização de diferentes desenhos de pesquisa e abordagens avaliativas.

Os resultados das avaliações mostraram que a participação no PAC ou no PAS impactou positivamente nos indicadores de saúde dos usuários e contribuiu para o aumento da prática de atividade física no lazer 3,32,36,40,43,44,45. Os benefícios da atividade física para a saúde são bem estabelecidos, os quais incluem a prevenção das DCNTs e dos seus fatores de risco, melhoria da saúde mental, interação social e aumento da resistência cardiorrespiratória e muscular 1,56. Nesse sentido, o PAC e o PAS promoveram e ampliaram as oportunidades para a prática de atividade física e fortaleceram as ações de promoção da saúde no Brasil 18. Esses programas também estão alinhados aos objetivos globais e à Agenda 2030, pois promovem a saúde e proporcionam o conhecimento da população sobre a importância e valorização da atividade física e outros hábitos saudáveis 56,57,58, além de criarem e manterem ambientes que favorecem os direitos da população, por permitirem o acesso equitativo a lugares e espaços para a prática de atividade física nas suas cidades e comunidades 56 . No entanto, o PAS e o PAC ainda apresentam desafios em relação ao planejamento, à organização, à oferta e à manutenção de atividades, bem como para a construção de novo polos, custeio e qualificação dos serviços ofertados. Somam-se ainda os desafios para a gestão em todos os níveis de governo, visto que são necessárias estratégias para evitar a devolução de recursos e garantir que os municípios tenham o programa em sua rede de serviços ${ }^{4}$. Esse cenário torna-se ainda mais agravado pelo avanço das políticas de austeridade fiscal e consequente congelamento de investimentos em saúde e indícios de desmontes de inúmeras políticas públicas. A falta de investimentos ameaça a continuidade e sustentabilidade dos programas comunitários de atividade física, além de potencializar o aumento das desigualdades sociais e piorar os indicadores de saúde 14,59 .

O estudo tem como limitações: os critérios de seleção dos marcos conceituais para a classificação das abordagens avaliativas foram baseados em autores clássicos do campo da avaliação, mas existem outras teorias e abordagens que podem ser aplicadas, porém essa escolha está relacionada também à subjetividade e à práxis do avaliador. Foram utilizadas para a seleção apenas quatro bases de dados, em contrapartida, consideraram-se aquelas que reúnem expressiva produção científica da área da saúde. As revisões frequentemente levam a uma busca mais ampla e menos definida, cujo objetivo é fornecer uma visão geral das evidências de pesquisa disponíveis sem produzir uma resposta resumida a uma questão de pesquisa discreta. Levando-se esse limite em consideração, precisaríamos de múltiplas pesquisas estruturadas para fornecer evidências mais precisas sobre os critérios escolhidos para este estudo: tipo de avaliação, indicadores e grau de inferência.

Como pontos fortes desta revisão pode-se considerar a variedade de estudos publicados nos últimos 12 anos sobre as avaliações feitas no PAC e no PAS, o que possibilitou identificar uma heterogeneidade de abordagens avaliativas, que utilizaram métodos quantitativos, qualitativos e misto, além de apresentar experiências de considerável destaque no país.

\section{Conclusão}

Foram incluídos nesta revisão 24 estudos publicados entre 2009 e 2020. As abordagens avaliativas foram referentes à avaliabilidade, à sustentabilidade, ao processo e ao resultado. As avaliações evidenciaram que o PAS e o PAC são estratégias efetivas de promoção da saúde e de incentivo à prática de atividade física no contexto do SUS, impactando positivamente nos indicadores de saúde dos usuários e no aumento da prática de atividade física no lazer. Os resultados permitem a ampliação da compreensão sobre as avaliações que foram realizadas no PAC e no PAS, fornecem elementos sobre as abordagens avaliativas que podem ser adaptadas em futuras avaliações, além de descreverem o 
panorama destes programas e do seu impacto para a promoção da saúde no Brasil. Reforçam também a importância da continuidade e investimentos nos programas comunitários de atividade física, especialmente em um cenário de instabilidade política e financeira que coloca em risco a sustentabilidade de programas e políticas sociais e de saúde.

Consideram-se fundamentais os estudos com revisões sistemáticas, meta-avaliação e novos estudos avaliativos, tanto locais quanto nacionais, que verifiquem a continuidade dos programas e das ações implementadas, a cobertura, o acesso, a efetividade e o impacto, bem como a avaliação da interferência do contexto político e econômico na prática de atividade física, na manutenção e investimento nos programas comunitários de atividade física, que têm se mostrado tão benéficos na promoção da saúde.

\section{Colaboradores}

A. G. Silva, E. J. S. Prates e D. C. Malta contribuíram na concepção e projeto do estudo, análise e interpretação dos dados, redação e revisão crítica do artigo e na aprovação final da versão a ser publicada; todos são responsáveis por todos os aspectos do trabalho na garantia da exatidão e integridade de qualquer parte da obra.

\section{Informações adicionais}

ORCID: Alanna Gomes da Silva (0000-0003-25875658); Elton Junio Sady Prates (0000-0002-5049186X); Deborah Carvalho Malta (0000-0002-82145734).

\section{Agradecimentos}

À Coordenação de Aperfeiçoamento de Pessoal de Nível Superior (CAPES) pela bolsa de doutorado de A. G. Silva; ao Fundo Nacional de Saúde do Ministério da Saúde pela bolsa de pesquisa de E. J. S. Prates; e ao Conselho Nacional de Desenvolvimento Científico e Tecnológico (CNPq) pela bolsa de produtividade de D. C. Malta.

\section{Referências}

1. Guthold R, Stevens GA, Riley LM, Bull FC. Worldwide trends in insufficient physical activity from 2001 to 2016: a pooled analysis of 358 population-based surveys with 1.9 million participants. Lancet Glob Health 2018; 6:e1077-86.

2. Mielke GI, da Silva ICM, Owen N, Hallal PC. Brazilian adults' sedentary behaviors by life domain: population-based study. PLoS One 2014; 9:e91614.

3. Hallal PC, Tenório MCM, Tassitano RM, Reis RS, Carvalho YM, Cruz DKA, et al. Avaliação do programa de promoção da atividade física Academia da Cidade de Recife, Pernambuco, Brasil: percepções de usuários e não-usuários. Cad Saúde Pública 2010; 26:70-8.

4. Sá GBAR, Dornelles GC, Cruz KG, Amorim RCA, Andrade SSCA, Oliveira TP, et al. O Programa Academia da Saúde como estratégia de promoção da saúde e modos de vida saudáveis: cenário nacional de implementação. Ciênc Saúde Colet 2016; 21:1849-60.

5. Lopes A, Ferreira A, Mendonça R, Dias MA, Rodrigues R, Santos L. Estratégia de promoção à saúde: Programa Academia da Cidade de Belo Horizonte. Rev Bras Ativ Fís Saúde 2016; $21: 379-84$ 
6. Ministério da Saúde. Política Nacional de Promoção da Saúde. http://bvsms.saude.gov. $\mathrm{br} / \mathrm{bvs} /$ publicacoes/politica_nacional_pro mocao_saude_pnaps.pdf (acessado em 09/ Nov/2020).

7. Malta DC, Silva MMA, Albuquerque GM, Amorim RCA, Rodrigues GBA, Silva TS, et al. Política Nacional de Promoção da Saúde, descrição da implementação do eixo atividade física e práticas corporais, 2006 a 2014. Rev Bras Ativ Fís Saúde 2014; 19:286-99.

8. Malta DC, Morais Neto OL, Silva MMA, Rocha D, Castro AM, Reis AAC, et al. National Health Promotion Policy (PNPS): chapters of a journey still under construction. Ciênc Saúde Colet 2016; 21:1683-94.

9. Ministério da Saúde. Portaria no 2.681, de 7 de novembro de 2013. Redefine o Programa Academia da Saúde no âmbito do Sistema Único de Saúde (SUS). Diário Oficial da União 2013; 8 nov.

10. Ministério da Saúde. Panorama nacional de implementação do Programa Academia da Saúde: monitoramento do Programa Academia da Saúde: ciclo 2017. http://bvsms.saude. gov.br/bvs/publicacoes/panorama_academia_ saude_monitoramento_programa.pdf (acessado em 21/Set/2020).

11. Malta DC, Castro AM, Gosch CS, Cruz DKA, Bressan A, Nogueira JD, et al. A Política Nacional de Promoção da Saúde e a agenda da atividade física no contexto do SUS. Epidemiol Serv Saúde 2009; 18:79-86.

12. Malta DC, Mielke GI, Costa NCP. Pesquisas de avaliação do Programa Academia da Saúde. Florianópolis: Sociedade Brasileira de Atividade Física e Saúde; 2020.

13. Fernandes AP, Andrade ACS, Costa DAS, Dias MAS, Malta DC, Caiaffa WT. Programa Academias da Saúde e a promoção da atividade física na cidade: a experiência de Belo Horizonte - MG, Brasil. Ciênc Saúde Colet 2017; 22:3903-14.

14. Crochemore-Silva I, Knuth AG, Mielke GI, Loch MR. Promoção de atividade física e as políticas públicas no combate às desigualdades: reflexões a partir da Lei dos Cuidados Inversos e Hipótese da Equidade Inversa. Cad Saúde Pública 2020; 36:e00155119.

15. Ministério da Saúde. Avaliação de efetividade de programas de atividade física no Brasil. http://bvsms.saude.gov.br/bvs/publicacoes/ avaliacao_efetividade_programas_atividade_ fisica.pdf (acessado em 09/Nov/2020).

16. Pedrosa JIS. Perspectivas na avaliação em promoção da saúde: uma abordagem institucional. Ciênc Saúde Colet 2004; 9:617-26.

17. Vieira-da-Silva LM, Furtado JP. A avaliação de programas de saúde: continuidades e mudanças. Cad Saúde Pública 2020; 36:e00237219.
18. Knuth AG, Simões EJ, Reis RS, Hallal PC, Cruz DKA, Zanchetta LM, et al. Avaliação de programas de atividade física no Brasil: uma revisão de evidências em experiências selecionadas. https://edisciplinas.usp.br/pluginfile. $\mathrm{php} / 372224 / \mathrm{mod}$ resource/content/1/Ava liaçãoMSBrasilProgramasdeAF.pdf (acessado em 09/Nov/2020).

19. Arksey H, O’Malley L. Scoping studies: towards a methodological framework. Int J Soc Res Methodol 2005; 8:19-32.

20. Tricco AC, Lillie E, Zarin W, O’Brien KK, Colquhoun H, Levac D, et al. PRISMA Extension for Scoping Reviews (PRISMA-ScR): checklist and explanation. Ann Intern Med 2018; 169:467-73.

21. The Joanna Briggs Institute. Joanna Briggs Institute reviewers' manual: 2014 edition. https://nursing.lsuhsc.edu/JBI/docs/Review ersManuals/Economic.pdf (acessado em 12/ Set/2020).

22. The Joanna Briggs Institute. JBI levels of evidence. https://joannabriggs.org/sites/ default/files/2019-05/JBI-Levels-of-evi dence_2014_0.pdf (acessado em 12/Set/2020).

23. Vieira-da-Silva LM. Avaliação de políticas e programas de saúde. Rio de Janeiro: Editora Fiocruz; 2018.

24. Pluye P, Potvin L, Denis JL, Pelletier J. Program sustainability: focus on organizational routines. Health Promot Int 2004; 19:489-500.

25. Habicht J, Victora CG, Vaughan JP. Evaluation designs for adequacy, plausibility and probability of public health programme performance and impact. Int J Epidemiol 1999; 28:10-8.

26. Donabedian A. Evaluating the quality of medical care. Milbank Q 2005; 83:691-729.

27. Padilha MA, Oliveira CM, Figueiró AC. Estudo de avaliabilidade do Programa Academia Carioca da Saúde: desafios para a promoção da saúde. Saúde Debate 2015; 39:375-86.

28. Silva RN, Guarda FRB, Hallal PC, Martelli PJL. Avaliabilidade do Programa Academia da Saúde no Município de Recife, Pernambuco, Brasil. Cad Saúde Pública 2017; 33:e00159415.

29. Paez DC, Reis RS, Parra DC, Hoehner CM, Sarmiento OL, Barros M, et al. Bridging the gap between research and practice: an assessment of external validity of community-based physical activity programs in Bogotá, Colombia, and Recife, Brazil. Transl Behav Med 2015; 5:1-11.

30. Cazarin G, Figueiró AC, Dias SF, Hartz Z. Análise da sustentabilidade de uma intervenção de promoção da saúde no Município de Recife - PE. Physis (Rio J.) 2019; 29:e290309. 
31. Mazo GZ, Quinaud PT, Salin MS, Virtuoso JF. Academias da saúde de Florianópolis: diferenças regionais na percepção dos idosos quanto aos serviços prestados, motivos de ingresso e permanência e nível de atividade física. ACM Arq Catarin Med 2013; 42:56-62.

32. Silva K, Sena R, Matos J, Lima K, Silva P. Acesso e utilização da Academia da Cidade de Belo Horizonte: perspectiva de usuários e monitores. Rev Bras Ativ Fís Saúde 2014; 19:700-10.

33. Ministério da Saúde. Monitoramento do Programa Academia da Saúde. http:// 189.28.128.100/dab/docs/portaldab/documen tos/devolutivas/Devolutiva-do-Monitora mento-Nacional-do-Programa-Academia-daSa--de-2015.pdf (acessado em 12/Set/2020).

34. Florindo AA, Nakamura PM, Farias Júnior JC, Siqueira FV, Reis RS, Cruz DKA, et al. Promoção da atividade física e da alimentação saudável e a saúde da família em municípios com academia da saúde. Rev Bras Educ Fís Esp 2016; 30:913-24.

35. Silva CRM, Bezerra J, Soares FC, Mota J, Barros MVG, Tassitano RM. Percepção de barreiras e facilitadores dos usuários para participação em programas de promoção da atividade física. Cad Saúde Pública 2020; 36:e00081019.

36. Hallal PC, Carvalho YM, Tassitano RM, Tenório MCM, Warschauer M, Reis RS, et al. Avaliação quali-quantitativa do programa academia da cidade, Recife - PE: concepções dos professores. Rev Bras Ativ Fís Saúde 2009; 14:9-14.

37. Simões EJ, Hallal P, Pratt M, Ramos L, Munk M, Damascena W, et al. Effects of a community-based, professionally supervised intervention on physical activity levels among residents of Recife, Brazil. Am J Public Health 2009; 99:68-75.

38. Mendonça BC, Oliveira AC, Toscano JJO, Knuth AG, Borges TT, Malta DC, et al. Exposure to a community-wide physical activity promotion program and leisure-time physical activity in Aracaju, Brazil. J Phys Act Health 2010; 7 Suppl 2:S223-8.

39. Fernandes AP, Andrade ACS, Ramos CGC, Friche AAL, Dias MAS, Xavier CC, et al. Leisure-time physical activity in the vicinity of Academias da Cidade Program in Belo Horizonte, Minas Gerais State, Brazil: the impact of a health promotion program on the community. Cad Saúde Pública 2015; 31 Suppl 1:S195207.

40. Feitosa W, Guarda F, Konrad L, Gonçalves W, Martelli P, Araújo Júnior J. Users' perception of actions, improvement in quality of life and satisfaction with the Academia da Cidade Program. Rev Bras Ativ Fís Saúde 2016; 21:461-9.
41. Simões EJ, Hallal PC, Siqueira FV, Schmaltz C, Menor D, Malta DC, et al. Effectiveness of a scaled up physical activity intervention in Brazil: a natural experiment. Prev Med 2017; 103S:S66-72.

42. Andrade ACS, Mingoti SA, Fernandes AP, Andrade RG, Friche AAL, Xavier CC, et al. Neighborhood-based physical activity differences: evaluation of the effect of health promotion program. PLoS One 2018; 13:e0192115.

43. Gonçalves LBB, Almeida RC, Oliveira TM, Palácio MAV, Pinto AGA. Programa academia da saúde: operacionalidade, ações e integração. Rev Bras Promoç Saúde 2019; 32:8381.

44. Maciel MG, Saraiva LAS, Silva MM, Vieira Junior PR. Avaliação de desempenho do programa academia da saúde em Belo Horizonte: um estudo de caso. Movimento (Porto Alegre) 2019; 25:e25026.

45. Melo EHR. Programas comunitários de atividade física: uma análise do Programa Academia da Cidade em Recife - PE [Dissertação de Mestrado]. Recife: Universidade Federal de Pernambuco; 2019.

46. Faria TMTR, Brenner S, Mielke GI. Avaliação do efeito do Programa Academia da Saúde sobre indicadores de comportamento saudável da população nas capitais brasileiras. In: Malta DC, Mielke GI, Costa NCP, organizadores. Pesquisas de avaliação do programa academia da saúde. Florianópolis: Sociedade Brasileira de Atividade Física e Saúde; 2020. p. 127-46.

47. Pratt M, Brownson RC, Ramos LR, Malta DC, Hallal PC, Reis RS, et al. Project GUIA: a model for understanding and promoting physical activity in Brazil and Latin America. J Phys Act Health 2010;7 Suppl 2:S131-4.

48. Malta DC, Reis AAC, Jaime PC, Morais Neto OL, Silva MMA, Akerman M. O SUS e a Política Nacional de Promoção da Saúde: perspectiva, resultados, avanços e desafios em tempos de crise. Ciênc Saúde Colet 2018; 23:1799809.

49. Malta DC, Silva MMA, Moura L, Morais Neto OL. A implantação do Sistema de Vigilância de Doenças Crônicas Não Transmissíveis no Brasil, 2003 a 2015: alcances e desafios. Rev Bras Epidemiol 2017; 20:661-75.

50. Vieira-da-Silva LM. Conceitos, abordagens e estratégias para a avaliação em saúde. In: Hartz ZMA, Vieira-da-Silva LM, organizadoras. Avaliação em saúde: dos modelos teóricos à prática na avaliação de programas e sistemas de saúde. Salvador: EDUFBA/Rio de Janeiro: Editora Fiocruz; 2005. p. 15-39. 
51. Medina MG, Silva GAP, Aquino R, Hartz ZMA. Uso de modelos teóricos na avaliação em saúde: aspectos conceituais e operacionais. In: Hartz ZMA, Vieira-da-Silva LM, organizadoras. Avaliação em saúde: dos modelos teóricos à prática na avaliação de programas e sistemas de saúde. Salvador: EDUFBA/Rio de Janeiro: Editora Fiocruz; 2005. p. 41-63.

52. Tanaka OY, Tamaki EM. O papel da avaliação para a tomada de decisão na gestão de serviços de saúde. Ciênc Saúde Colet 2012; 17:821-8.

53. Contandriopoulos AP. Avaliando a institucionalização da avaliação. Ciênc Saúde Colet 2006; 11:705-11.

54. Ramos MC, Silva EN. Como usar a abordagem da política informada por evidência na saúde pública? Saúde Debate 2018; 42:296-306.

55. Silva LMV, Formigli VLA. Avaliação em saúde: limites e perspectivas. Cad Saúde Pública 1994; 10:80-91.

56. World Health Organization. Global action plan on physical activity 2018-2030: more active people for a healthier world. https://apps.who.int/iris/bitstream/hand le/10665/272722/9789241514187-eng.pdf (acessado em 12/Set/2020).
57. Organização das Nações Unidas. Agenda 2030. Os 17 Objetivos de Desenvolvimento Sustentável. http://www.agenda2030.org.br/ods/3/ (acessado em 06/Nov/2020).

58. World Health Organization. Global action plan for the prevention and control of noncommunicable diseases 2013-2020. Geneva: World Health Organization; 2013.

59. Malta DC, Duncan BB, Barros MBA, Katikireddi SV, Souza FM, Silva AG, et al. Medidas de austeridade fiscal comprometem metas de controle de doenças não transmissíveis no Brasil. Ciênc Saúde Colet 2018; 23:3115-22.

60. Moher D, Liberati A, Tetzlaff J, Altman DG. Preferred Reporting Items for Systematic Reviews and Meta-Analyses: the PRISMA statement. PLoS Med 2009; 6:e1000097. 


\section{Abstract}

Community physical activity programs were created to encourage and increase the practice of physical activity in the Brazilian population and promote healthy life habits. The Brazilian Ministry of Health invested in the evaluation of these programs and consolidated partnerships that favor the development of relevant highlights on the topic. The current study aimed to identify and summarize the scientific evidence on the approaches and results of evaluations performed in the Health Academy Program and City Academy Program. This is a scoping review based on the methodology of the Joanna Briggs Institute. We used the MEDLINE via PubMed, LILACS, Scopus, and Cochrane databases, the website of the Health Academy Program, the Catalogue of Theses and Dissertations of the Brazilian Graduate Studies Coordinating Board, and the Brazilian Digital Library of Theses and Dissertations. The sample included quantitative or qualitative primary studies with no limit on year of publication. Twentyfour studies published from 2009 to 2020 were selected and subdivided according to the approaches to evaluation: evaluability, sustainability, process (supply and structure), outcome (impact and satisfaction), and degree of inference (adequacy, plausibility, and probability). The results of the evaluations showed that the programs offer various activities, positively impact users' health indicators, and contribute to the increase in leisure-time physical activity. The evaluation of these programs is essential for the administration, health services, and healthcare workers, since it allows verifying the implementation of the proposed activities, coverage, access, impact, and interference by the political context in their continuity.

Program Evaluation; Health Promotion; Motor Activity; Review

\section{Resumen}

Los programas comunitarios de actividad fisica fueron creados para incentivar y aumentar la práctica de actividad física en la población brasileña y promover hábitos de vida saludable. El Ministerio de Salud de Brasil invirtió en la evaluación de estos programas y consolidó colaboraciones que favorecieron la construcción de importantes evidencias sobre este asunto. Este estudio tuvo el objetivo de identificar y sintetizar las evidencias científicas sobre los abordajes y resultados de las evaluaciones realizadas en el Programa Academia de la Salud y Programa Academia de la Ciudad. Se trata de una revisión de alcance, basada en la metodología del Instituto Joanna Briggs. Se utilizaron las bases MEDLINE a través de PubMed, LILACS, Scopus y Cochrane, el sitio web del Programa Academia de la Salud, el Catálogo de Tesis y Disertaciones de la Coordinación de Perfeccionamiento de Personal de Nivel Superior y en la Biblioteca Digital Brasileña de Tesis $y$ Disertaciones. Se incluyeron estudios primarios cuantitativos o cualitativos, sin limite temporal. Se seleccionaron 24 publicaciones entre $2009 y$ 2020. Se subdividieron de acuerdo con los abordajes de evaluación: disponibilidad, sostenibilidad, proceso (oferta y estructura), resultado (impacto y satisfacción) y grado de inferencia (adecuación, plausibilidad y probabilidad). Los resultados de las evaluaciones mostraron que los programas ofrecen diversas actividades, impactan positivamente en los indicadores de salud de los usuarios y contribuyen al aumento de actividad física en el ocio. La evaluación de estos programas es fundamental para la gestión, servicios de salud y profesionales, puesto que permite verificar la implementación de las acciones propuestas, la cobertura, el acceso y el impacto, así como la interferencia del contexto político en su continuidad.

Evaluación de Programas y Proyectos de Salud; Promoción de la Salud; Actividad Motora; Revisión
Recebido em 21/Set/2020

Versão final reapresentada em 26/Dez/2020

Aprovado em 04/Jan/2021 